\title{
Asphaltene Molecular Representation: Impact on Aggregation Evaluation
}

\author{
Ana Milena Velásquez, Jennifer de León, Bibian A. Hoyos \\ Universidad Nacional de Colombia-Sede Medellín, Facultad de Minas, Departamento de Procesos y Energía \\ Carrera 80 No. 65-223, Núcleo Robledo, Medellín, Colombia \\ amvelasquezv@unal.edu.co; jde@unal.edu.co; bahoyos@unal.edu.co
}

\begin{abstract}
Molecular dynamics simulations were used to evaluate the effect of the asphaltene molecular representation on calculations of the aggregate size and aggregation behavior of asphaltene/solvent systems. Three different asphaltene representations were studied, namely, a mixture of four molecules, an island-type molecule and an archipelago-type molecule. Calculations were conducted for pure asphaltene systems and in solutions of $n$-heptane and toluene. For pure asphaltene systems, the island-type representation allows for the formation of extremely large aggregates, whereas for the mixture and archipelago representations, the aggregates contained up to four molecules. For asphaltene/solvent systems, the mixture representation was consistent with the expected solubility behavior of asphaltenes in both n-heptane and toluene. With this representation, the final configuration in n-heptane consisted of up to fourmolecule aggregates, whereas in toluene, the observed aggregates were dimers, at most. The structural configuration of the island-type molecule misrepresented the aggregation behavior of the asphaltenic phase. The representation of the asphaltene phase, exclusively with the archipelago architecture, also fails to correctly describe the asphaltene aggregation since almost no aggregation was observed. In n-heptane, the asphaltene aggregates were compact and stable with time, and their behavior resembled that of solid particles suspended in a fluid phase. In toluene, the aggregates were of a porous nature, forming viscoelastic networks and reducing the mobility of the fluid phase. The results indicate that the mixture representation is a more appropriate choice for the evaluation of asphaltenic system behavior.
\end{abstract}

Keywords: Asphaltene, molecular simulation, aggregation

\section{Introduction}

Asphaltenes are defined as the heaviest fraction of crude oil, insoluble in paraffinic hydrocarbons, such as pentane or heptane, and soluble in aromatic compounds, such as toluene. Asphaltenes tend to form large aggregates that can generate a sudden increase in oil viscosity and can reduce fluid mobility. Understanding the phenomenology behind asphaltene aggregation and the evolution of aggregate size brings the industry one-step closer to designing appropriate strategies to increase oil recovery.

There are two distinct models aimed at describing asphaltene aggregation behavior. On the one hand, the Yen-Mullins model [1] presents a hierarchical aggregation description, in which asphaltenes are represented by a single molecule with one aromatic core (with four to ten aromatic rings) and aliphatic chains attached to the periphery. In this island-type architecture, $\pi-\pi$ interactions between the aromatic cores of asphaltene molecules are the main force behind the aggregation process. The Yen-Mullins model has been successfully used in molecular simulations to reproduce experimental results, such as intermolecular distances in aggregates [2], aggregate size [3] and solubility behavior [4]. However, this model is inadequate in explaining the ability of asphaltene aggregates to host other molecules within them (solvent molecules [5], metallic porphyrins [6] and so on), and the strong adsorption interactions observed between asphaltene aggregates and different types of surfaces, such as formation rock, production pipes and surface equipment [7], [8].

The supramolecular model, on the other hand, considers asphaltenes as a large family of structures, and asphaltene aggregation as a more complex phenomenon, in which not only $\pi-\pi$ interactions exist, but where acid-base interactions, hydrogen bonding, metallic interactions, and interactions between alkylic and cycloalkylic groups must also be considered [8], [9]. Aggregation is then considered as a consequence of the simultaneous occurrence of all these interactions. This model is an attempt to explain the behavior of asphaltene aggregates in different solvents, different interactions with surfaces and the formation of flexible and porous aggregates. 
Molecular simulation studies have been conducted previously in order to reproduce and ultimately predict asphaltene aggregation behavior. Aggregation of island-type molecules in a vacuum [10] and in non-explicit solvents, represented by their dielectric constant [11], prove that the separation distance between aromatic cores in asphaltene aggregates is between 3 and $4 \AA$, and that the spatial configuration of dimers can be face-to-face or parallel $(\pi-\pi)$, offset stacking or offset parallel $(\sigma-\sigma)$, and edge-to-face or T-shape $(\pi-\sigma)[12]$. Increases in computational capacity has allowed the solvent to be described in an explicit way, proving that the aggregation in solvents is similar to the aggregation in a vacuum [13], [14].

Different molecular simulation studies have been published, utilizing different metrics to quantify the evolution of the average aggregate size throughout the simulation time [3], [12], but none containing a detailed analysis of the effect of the molecular structure and the concentration of the asphaltenes on the average aggregate size.

Recent publications show that the overall behavior of asphaltene molecules and aggregates is affected by the nature of the solvent. The addition of $n$-heptane, for instance, generates an increase in the aggregate size of asphaltenic systems [15], [16], whereas the addition of toluene has no noticeable impact on this variable.

The aim of this work is to evaluate the effect of the molecular representation of the asphaltene fraction on the average aggregate size calculations of asphaltene/solvent systems using molecular dynamics simulations. Asphaltene aggregation was studied in a vacuum and in solvents of a paraffinic (n-heptane) and an aromatic nature (toluene) at concentrations of 18.6 and $30.0 \mathrm{wt} \%$. We evaluated three different asphaltene representations, namely, a mixture of four molecules and systems with the asphaltene phase represented exclusively with island-type molecules or archipelago-type molecules.

\section{Model}

The structure of asphaltene molecules used in this study was constructed by an stochastic algorithm that generates structures from experimental data of asphaltene samples [17]. This algorithm specifically avoids the construction of molecules with the pentane effect [18] and violation of the Clar's sextet rule for the amount and location of disjoint aromatic $\pi$-sextets in the core of asphaltene molecules [19].

Figure 1 depicts the asphaltene molecular representations obtained from the stochastic algorithm, with their corresponding molecular weights and the fraction they have in the mixture. These representations were generated from the experimental data of Maya crude oil [20], [21]. It is worth noting that all four molecules, as individuals, as well as the pondered average of the set, are within the accepted criteria for the asphaltene structural description [22]. In addition, all the molecules are free from the pentane effect and abide the Clar's sextet rule.
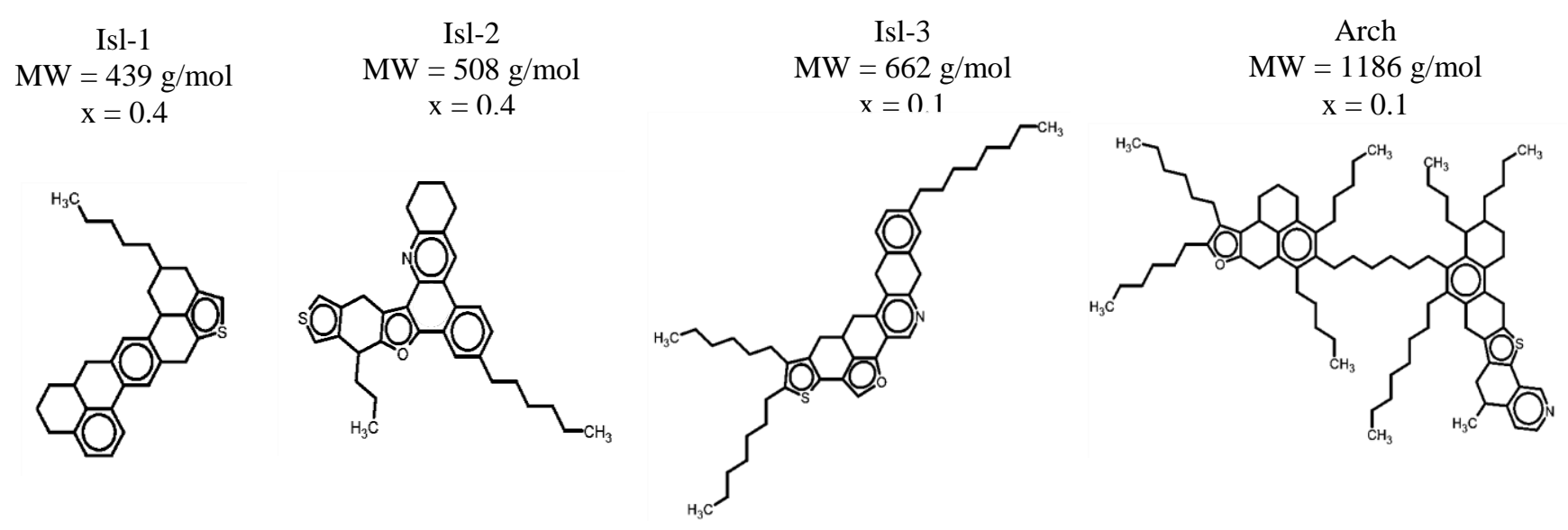

Fig. 1: Molecular representations of asphaltenes generated for Maya crude oil.

From Figure 1, the generated molecular representations include three island-type molecules, two with a continental structure (labeled as Isl-1 and Isl-2) and a third one with a rosary-type configuration (Isl-3), all three accounting for $90 \%$ of the asphaltene phase. The remaining $10 \%$ of the asphaltene phase is an archipelago-type molecule (Arch). Molecule Isl-1 has a single aliphatic chain and has sulfur as its only heteroatom. Molecule Isl-2 has two aliphatic substitutions and three heteroatoms (sulfur, nitrogen and oxygen). Molecule Isl-3 has the same 
heteroatoms as Isl-2, but has an additional aliphatic substitution. Note that the molecular weight of the island-type molecules is of the same order of magnitude, but increases continuously from Isl-1 to Isl-3. Molecule Arch has two aromatic units in its structure, linked by an alkyl bridge of six carbons. The molecular weight of this archipelago-type structure is around twice that of Isl-2 or Isl-3, but is still within the accepted criteria for the molecular weight of asphaltene molecules. The different architectures and numbers of heteroatoms present in these model molecules allows us to determine the impact of the molecular structure of asphaltenes on the size of the aggregates in n-heptane and toluene solutions.

In order to quantify the average aggregate size, the average number of asphaltene molecules forming the aggregates, $\langle m\rangle$, was used (Eq. 1):

$$
\langle m\rangle=\frac{\sum m N_{m}}{\sum N_{m}}
$$

where $N_{m}$ is the number of aggregates containing $m$ asphaltene molecules. This evaluation of the average size has been used in previous research [3].

As aggregation criteria, two molecules were considered aggregated when there was a separation of $4 \AA$ or less between their aromatic cores. This value is comparable with experimental reports that indicate that the distance between aromatic planes of aggregated asphaltene molecules is between 3.6 and 3.8 $\AA$ [23], and it has been successfully used in aggregation studies using molecular simulation techniques [12].

Figure 2 shows a schematic of the measurement procedure of the distance between the aromatic cores of aggregated asphaltene molecules. As can be seen from the figure, all measurements are below $4 \AA$, and thus, these molecules form a four-molecule aggregate.

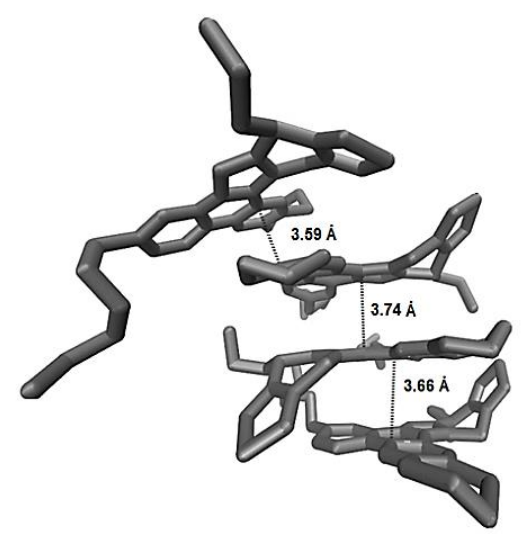

Fig. 2: Schematic of the measurement of the distance between aromatic planes of aggregated asphaltene molecules.

The aggregation study was conducted for systems in a vacuum (absence of solvent) and in systems with asphaltene concentrations of 18.6 and $30.0 \mathrm{wt} . \%$ in n-heptane and toluene.

For the aggregation study of pure asphaltene systems, we evaluated three different representations, a system consisting of the mixture presented in Figure 1, with the proportions shown there, and systems with the asphaltene phase represented exclusively with Isl-1 or Arch molecules, respectively. With these three systems (a mixture, an island-type representation and an archipelago-type representation), the effect of the molecular structure of asphaltenes on the aggregate size can be evaluated. For these calculations, 20 to 36 asphaltene molecules were placed in the simulation box.

For calculations with solvents, three asphaltene systems were also used (mixture, Isl-1 and Arch), using 10 asphaltene molecules in all cases. For the simulations with n-heptane, between 102 and 517 heptane molecules were simulated (depending on the asphaltene concentration). In the case of toluene, 111 to 562 solvent molecules were used.

Before the simulations were carried out, the intramolecular energy of a single molecule of each compound was minimized by adjusting the atomic coordinates of each atom iteratively until convergence was achieved at low energy [24]. At the beginning of every simulation, asphaltene molecules were placed at a distance of $12 \AA$ from each other, and when 
necessary, the respective solvent molecules were placed around them. This initial distance was set so that each asphaltene molecule was within the cutoff radius for the long-range interactions with the neighboring asphalthene molecules, but at a distance large enough that the aggregation criteria were not fulfilled.

An initial energetic relaxation of each system was made in an NVT ensemble for $1 \mathrm{~ns}$, by placing the molecules in a volume large enough to guarantee low density and avoid molecular overlapping $(0.01-0.10 \mathrm{~g} / \mathrm{ml})$. Then, a simulation with an NPT ensemble at $1 \mathrm{~atm}$ was carried out for $1 \mathrm{~ns}$ to reach a stable value for the volume of the simulation box. This volume was then set for an additional NVT simulation for $30 \mathrm{~ns}$, in order to evaluate the time evolution of aggregation size. The time step was set to $1 \mathrm{fs}$ in the equilibration stages, and to $2 \mathrm{fs}$ in the long NVT simulations of aggregation evaluation.

The consistent valence force field (CVFF) potential [25] was used to describe asphaltene and solvent molecules, in which all atoms are considered explicitly (all-atom potential). This potential considers Van der Waals and electrostatic intermolecular interactions, as well as intramolecular bond, angles and dihedral interactions [26]. Partial charges for the atoms in each molecule were calculated using the equivalent charges method [27], and long-range interactions were evaluated with the particle-particle particle-mesh (pppm) technique, with a cutoff radius of $16 \AA$. The SHAKE algorithm was used to maintain bond lengths and angles during the simulations. All simulations were conducted at $298 \mathrm{~K}$, and for the NPT simulations, the pressure was set to $1 \mathrm{~atm}$, controlled by the Nosé-Hoover thermostat and barostat, respectively. Periodic boundary conditions were used in all three directions. The large-scale atomic/molecular massively parallel simulator (LAMMPS) [26] was used for molecular dynamics calculations.

\section{Results}

Figure 3 shows the time evolution of the average aggregate size, $\langle m\rangle$, for the three pure asphaltene representations. For the Arch and Isl-1 representations, the systems exhibit an increasing aggregation behavior. The largest aggregate size was obtained with the Isl-1 representation, with a final average aggregate size of 3.3 after $30 \mathrm{~ns}$. This average size results from a final configuration of one large aggregate of 22 asphaltene molecules, seven molecules forming small aggregates of two or three molecules, and the remaining seven molecules in a monomeric state. For the Arch representation, a final average size of 3.0 is reached, associated with aggregates consisting of two to four asphaltenes. For the mixture system, an average size of 2.9 was reached at the early stages of the simulation, and remained constant until the end. This average size is consistent with aggregates of two to four molecules, similar to the Arch representation.

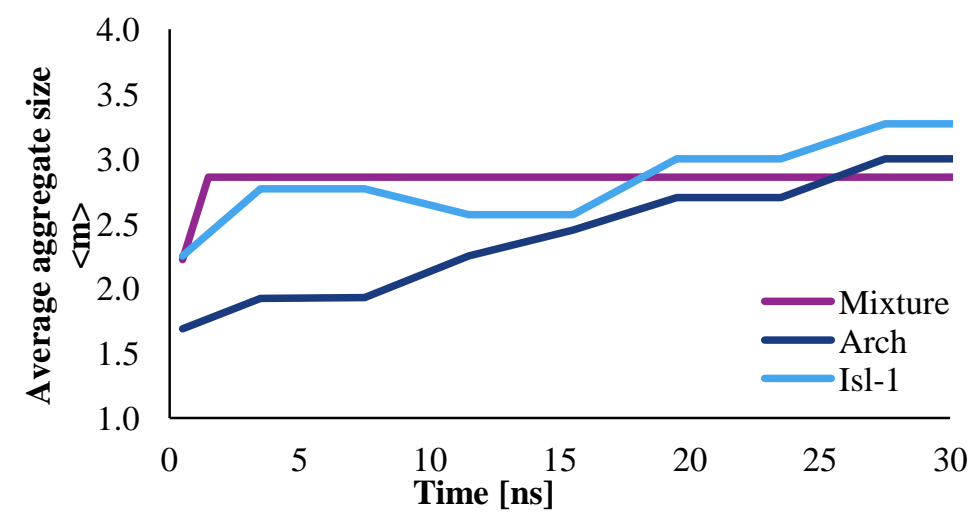

Fig. 3: Average aggregate size for the three pure asphaltene systems.

By analyzing only the average aggregate size of pure asphaltenes, it seems that the aggregation behavior is independent of the molecular representation used for the asphaltene phase. For asphaltenes in a vacuum, the average aggregate size was $\sim 3$ for all cases. However, when comparing the final configurations of the three systems, it was clear that the aggregation behavior obtained with the Isl-1 representation was far from the other two cases. The large aggregate of 22 asphaltene molecules obtained with this representation was possibly due to the molecular structure of the Isl-1 asphaltene, with one short aliphatic substitution, which decreases steric hindrance and facilitates aggregation. With the Arch and mixture representations, the multiple ramifications of the asphaltene molecules create steric impedances that stop 
aggregation beyond four-molecule aggregates. The analysis of the average aggregate size must be complemented in each case with the final configuration description for an accurate explanation of the aggregation process.

Figure 4 depicts the average aggregation size for all three representations of the asphaltene phase with a concentration of $30.0 \mathrm{wt} . \%$ in $\mathrm{n}$-heptane. It can be seen that the largest average size was obtained for the mixture representation, with an average size of 2.0. This is a result of a final configuration of one four-molecule aggregate, one three-molecule aggregate and the remaining molecules in a monomeric state. For the Isl-1 and Arch representations, average sizes of 1.3 and 1.1 were obtained, respectively. The result for Isl-1 is consistent with two two-molecule aggregates and the remaining molecules in a monomeric state, whereas with the Arch representation, there was only a dimer.

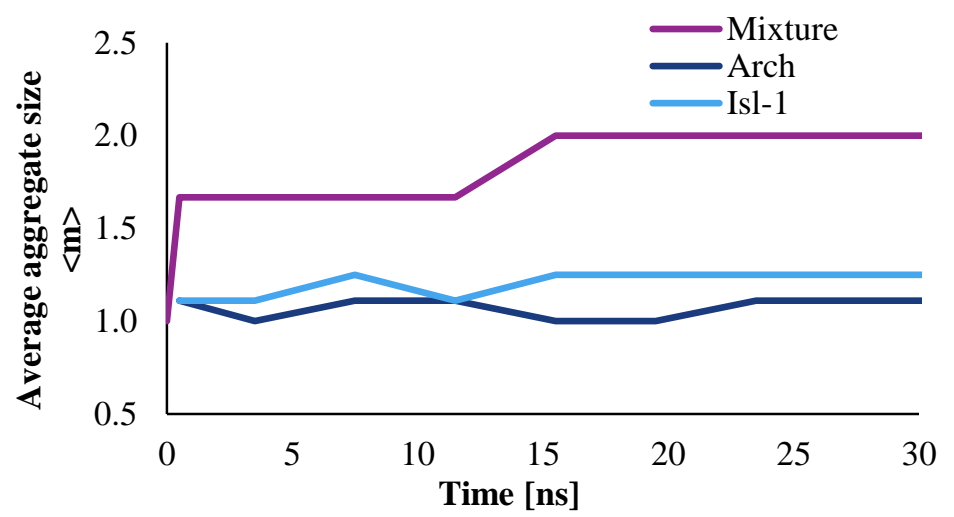

Fig. 4: Average aggregate size for the three asphaltene representations at $30.0 \mathrm{wt} . \% \mathrm{in} n$-heptane.

Figure 5 shows the average aggregate size for the three asphaltene representations at a concentration of 18.6 wt. $\%$ in $\mathrm{n}$-heptane. As can be seen from the figure, the largest average size was obtained for the mixture representation, similar to the results for the $30.0 \mathrm{wt}$.\% concentration. In this case, the average size was between 2.0 and 2.5, whereas for the Isl- 1 and Arch representations, the average aggregate size was significantly lower. For the Arch representation, the average size was 1.0, which is equivalent to all the asphaltene molecules being in a monomeric state.

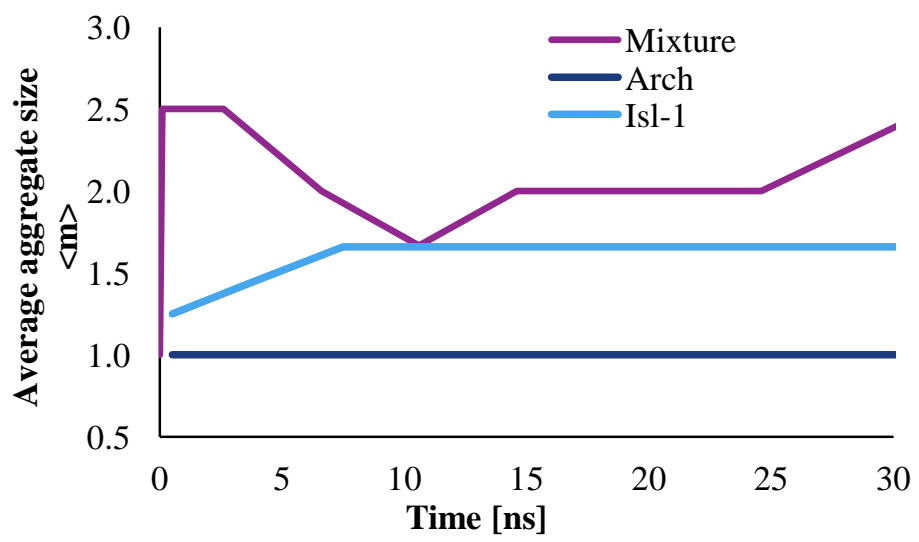

Fig. 5: Average aggregate size for the three asphaltene representations at $18.6 \mathrm{wt} . \%$ in $\mathrm{n}$-heptane.

The simulations for asphaltenes represented as the mixture in n-heptane, for the two concentrations studied here, were extended until $95 \mathrm{~ns}$ of simulation time (not shown), and in both cases, the average aggregate size was 2.0. Aggregates of more than four molecules are temporarily formed, but they dissolve as the simulation continues. For this representation, aggregates up to four molecules are stable for the entire simulation time. Additional molecules can eventually interact with the four-molecule aggregates, even below the aggregation distance. However, steric hindrance due to the multiple chains cause these large aggregates to be unstable, and thus the attached molecules cannot remain part of the aggregate.

Although for the mixture representation, the final average size is the same for both concentrations in $\mathrm{n}$-heptane. For the lower concentration, the final configuration consisted of two- or three-molecule asphaltene aggregates, whereas for the 
30.0 wt.\% system, the final configuration consisted of three- and four-molecule aggregates. This indicates that for the mixture representation in $n$-heptane, the aggregate size increases with asphaltene concentration, as expected for asphaltenes in a paraffinic solvent [28], [29]. The growth of asphaltene aggregates is limited to a few molecules due to steric repulsion (four-molecule aggregates).

For the Isl-1 representation, the formation of aggregates of fewer molecules in the presence of n-heptane, compared with the pure asphaltene behavior presented in Figure 3, is due to the interaction between asphaltene and n-heptane molecules, which weakens the attractive forces between the relatively small aromatic cores of these asphaltenes.

With the Arch representation, practically no aggregation was observed for the two concentrations of asphaltenes in nheptane. The interaction between the aliphatic chains in these asphaltene molecules and the paraffinic hydrocarbon in the solvent, create enough separation between aromatic cores for the molecules to exceed the aggregation criteria. This, however, is inconsistent with the known asphaltene aggregation behavior in n-heptane, meaning that the representation of the asphaltene phase exclusively with the archipelago architecture fails to correctly describe the aggregation behavior of these species.

Figure 6 presents the average aggregate size for the three representations of the asphaltene phase at concentrations of 18.6 and $30.0 \mathrm{wt} \%$ in toluene. For both concentrations, the mixture representation reaches an average size higher than for the Isl-1 and Arch representations. However, as the simulation advances, all three representations reach an average size between 1.0 and 1.5. The simulations with the mixture representation were extended until 95 ns (not shown) and the average size remained within this range. This is consistent with two two-molecule aggregates at most, and the remaining asphaltene molecules in the monomeric state.

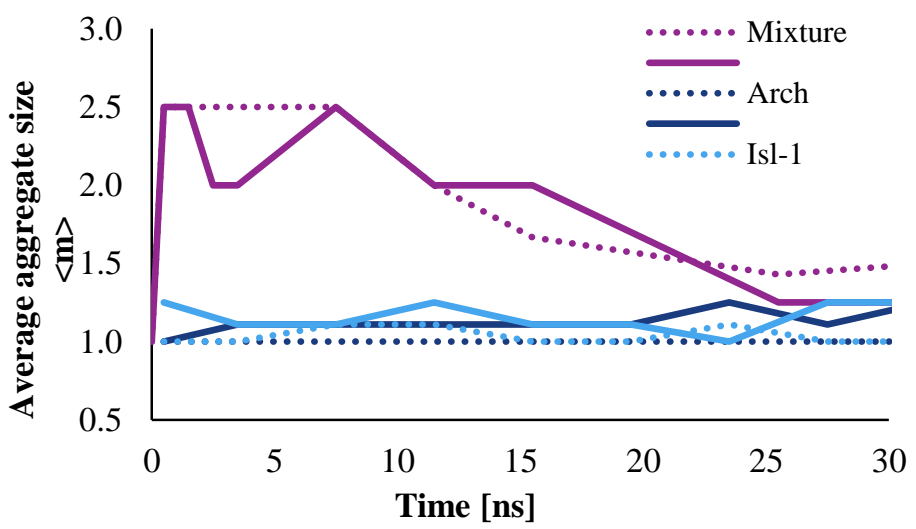

Fig. 6: Average aggregate size for the three asphaltene representations at 18.6 (dotted line) and $30.0 \mathrm{wt} . \%$ (solid line) in toluene.

From Figure 6, the aggregation behavior of asphaltenes in toluene was almost independent of the concentration for all three cases. The aromatic nature of the solvent promotes the interaction between solvent and asphaltene molecules, neutralizing the active points in the asphaltenes and decreasing the average aggregate size.

From the results of average aggregate size, the mixture representation for the asphaltene phase gives the best overall representation for both concentrations. The use of this representation of four molecules does not imply a significant increase in computational time compared with the representations with a single molecule, but allows for further research regarding asphaltene adsorption on different types of surfaces, aggregate structure and porosity, and so on.

\section{Conclusions}

In this work, the effect of the molecular representation of the asphaltene phase on the aggregate size calculations of asphaltene/solvent systems were evaluated. Three molecular representations were used for the asphaltene phase, namely, a mixture of four molecules, and two representations consisting of either island-type or archipelago-type molecules.

The average aggregate size for pure asphaltene systems was independent to the molecular representation used. However, for the island-type representation, the presence of short aliphatic chains allows for the formation of extremely large aggregates in a vacuum, whereas for the mixture and archipelago representations, the long aliphatic chains increase steric hindrance and stop the growth of asphaltene aggregates, limiting the size of the aggregates to up to four molecules. 
For asphaltene/solvent systems, aggregation results with the mixture representation were consistent with the expected solubility behavior of asphaltenes in both n-heptane and toluene. With this representation, the final configuration in nheptane consisted of up to four-molecule aggregates, whereas in toluene, the aggregates were dimers at most.

For the island and archipelago representations, the average aggregate size was significantly lower than with the mixture representation. The structural configuration of the island-type molecule selected misrepresented the aggregation behavior of the asphaltenic phase. The representation of the asphaltene phase exclusively with the archipelago architecture also fails to correctly describe the asphaltene aggregation since practically no aggregation was observed.

Finally, the results of aggregation indicate that the mixture representation is a more appropriate choice for the evaluation of the behavior of asphaltenic systems. In addition, by having both island-type and archipelago-type structures, the mixture representation of the asphaltene phase allows the study of different scenarios for asphaltenic systems. Future

research should include adsorption of asphaltene aggregates on different types of surfaces and the porosity of the aggregates.

\section{Acknowledgements}

Financial support for this work was provided by Agencia Nacional de Hidrocarburos (ANH) and Colciencias (call for proposals 721-2015, project 111872150012, Contract No. FP44842-016-2016). The authors also thank the Universidad Nacional de Colombia- Sede Medellín for allowing simulations in the advanced numerical computation unit (UNICA).

\section{References}

[1] O. C. Mullins, “The Modified Yen Model," Energy \& Fuels, vol. 24, no. 4, pp. 2179-2207, 2010.

[2] E. Rogel, "Studies on asphaltene aggregation via computational chemistry," Colloids Surfaces A Physicochem. Eng. Asp., vol. 104, no. 1, pp. 85-93, 1995.

[3] P. Ungerer, D. Rigby, B. Leblanc, and M. Yiannourakou, "Sensitivity of the aggregation behaviour of asphaltenes to molecular weight and structure using molecular dynamics," Mol. Simul., vol. 40, no. 1-3, pp. 115-122, 2014.

[4] E. Rogel, C. Ovalles, and M. Moir, "Asphaltene Chemical Characterization as a Function of Solubility: Effects on Stability and Aggregation," Energy \& Fuels, vol. 26, no. 5, pp. 2655-2662, 2012.

[5] M. Derakhshesh, A. Bergmann, and M. R. Gray, "Occlusion of Polyaromatic Compounds in Asphaltene Precipitates Suggests Porous Nanoaggregates," Energy \& Fuels, vol. 27, no. 4, pp. 1748-1751, 2013.

[6] S. Acevedo, K. Guzmán, H. Labrador, H. Carrier, B. Bouyssiere, and R. Lobinski, "Trapping of Metallic Porphyrins by Asphaltene Aggregates: A Size Exclusion Microchromatography With High-Resolution Inductively Coupled Plasma Mass Spectrometric Detection Study," Energy \& Fuels, vol. 26, no. 8, pp. 4968-4977, 2012.

[7] J. S. Buckley, “Asphaltene Deposition,” Energy \& Fuels, vol. 26, pp. 4086-4090, 2012.

[8] M. R. Gray, R. R. Tykwinski, J. M. Stryker, and X. Tan, "Supramolecular Assembly Model for Aggregation of Petroleum Asphaltenes," Energy \& Fuels, vol. 25, no. 7, pp. 3125-3134, 2011.

[9] A. Z. Tukhvatullina, E. E. Barskaya, V. N. Kouryakov, T. N. Yusupova, and G. V Romanov, "Supramolecular Structures of Oil Systems as the Key to Regulation of Oil Behavior," J. Pet. Environ. Biotechnol., vol. 4, no. 4, pp. $1-8,2013$.

[10] J. H. Pacheco Sánchez, I. P. Zaragoza, and J. M. Martinez-Magadán, "Asphaltene Aggregation under Vacuum at Different Temperatures by Molecular Dynamics," Energy \& Fuels, vol. 17, no. 5, pp. 1346-1355, 2003.

[11] A. Ortega-Rodríguez, S. A. Cruz, A. Gil-Villegas, F. Guevara-Rodríguez, and C. Lira-Galeana, "Molecular View of the Asphaltene Aggregation Behavior in Asphaltene - Resin Mixtures," Energy \& Fuels, vol. 17, pp. 1100-1108, 2003.

[12] M. Sedghi, L. Goual, W. Welch, and J. Kubelka, "Effect of asphaltene structure on association and aggregation using molecular dynamics," J. Phys. Chem. B, vol. 117, no. 18, pp. 5765-76, 2013.

[13] T. F. Headen and E. S. Boek, "Molecular Dynamics Simulations of Asphaltene Aggregation in Supercritical Carbon Dioxide with and without Limonene," Energy \& Fuels, vol. 25, no. 2, pp. 503-508, 2011.

[14] J. H. Pacheco-Sáncez, F. Álvarez-Ramírez, and J. M. Martínez-Magadán, "Morphology of Aggregated Asphaltene Structural Models," Energy \& Fuels, vol. 18, pp. 1676-1686, 2004.

[15] L. Barré, "Solution Properties of Asphaltenes," Langmuir, vol. 24, no. 923579287, pp. 3709-3717, 2008.

[16] J. L. Mendoza de la Cruz, J. C. Cedillo-Ramírez, A. D. J. Aguirre-Gutiérrez, F. García-Sánchez, and M. A. AquinoOlivos, "Incompatibility Determination of Crude Oil Blends from Experimental Viscosity and Density Data," 
Energy \& Fuels, p. $150116110854002,2015$.

[17] J. De León and B. A. Hoyos, "A stochastic method for asphaltene structure formulation from experimental data: Avoidance of implausible structures," in The Sixteenth International Conference on Petroleum Phase Behavior and Fouling., pp. 1-10, 2015.

[18] D. D. Li and M. L. Greenfield, "High Internal Energies of Proposed Asphaltene Structures," Energy \& Fuels, vol. 25, pp. 3698-3705, 2011.

[19] F. J. Martín-Martínez, E. H. Fini, and M. J. Buehler, "Molecular asphaltene models based on Clar sextet theory," RSC Adv., vol. 5, no. 1, pp. 753-759, 2015.

[20] T. Takanohashi, S. Sato, and R. Tanaka, "Structural Relaxation Behaviors of Three Different Asphaltenes Using MD Calculations," Pet. Sci. Technol., vol. 22, no. 7-8, pp. 901-914, 2004.

[21] W. Tang, M. R. Hurt, H. Sheng, J. Riedeman, D. J. Borton, and H. I. Kenttämaa, "Structural Comparison of Asphaltenes of Different Origins by Using Tandem Mass Spectrometry,” Energy, vol. 29, pp. 1309-1314, 2015.

[22] O. C. Mullins, H. Sabbah, A. E. Pomerantz, A. B. Andrews, Y. Ruiz-morales, F. Mostow, R. Mcfarlane, L. Goual, R. Lepkowicz, T. Cooper, J. Orbulescu, R. M. Leblanc, J. Edwards, and R. N. Zare, "Advances in Asphaltene Science and the Yen - Mullins Model," Energy \& Fuels, vol. 26, pp. 3986-4003, 2012.

[23] S. S. Pollack and T. F. Yen, "Structural Studies of Asphaltics by X-Ray Small Angle Scattering," Anal. Chemestry, vol. 42, no. 6, pp. 623-629, 1970.

[24] J. Nocedal and S. J. Wright, Numerical Optimization, Second Edi. Verlag New York: Springer, 1999.

[25] M. Levitt and M. Hirshberg, "Potential energy function and parameters for simulations of the molecular dynamics of proteins and nucleic acids in solution," Comput. Phys. Commun., vol. 91, no. 1-3, pp. 215-231, 1994.

[26] S. Plimpton, "Fast Parallel Algorithms for Short-Range Molecular Dynamics," Journal of Computational Physics, vol. 117, no. 1. pp. 1-19, 1995.

[27] A. K. Rappé and W. A. Goddard III, "Charge Equilibration for Molecular Dynamics Simulations," J. Phys. Chem., vol. 95 , no. 8 , pp. 3358-3363, 1991.

[28] Z. Wang, J. Hu, H. Shui, S. Ren, C. Wei, C. Pan, Z. Lei, and X. Cui, "Study on the structure and association of asphaltene derived from liquefaction of lignite by fluorescence spectroscopy," Fuel, vol. 109, pp. 94-100, 2013.

[29] C. Jian, T. Tang, and S. Bhattacharjee, "Probing the Effect of Side-Chain Length on the Aggregation of a Model Asphaltene Using Molecular Dynamics Simulations," Energy \& Fuels, vol. 27, pp. 2057-2067, 2013. 